\title{
Implementation of universal quantum gates based on nonadiabatic geometric phases
}

\author{
Shi-Liang Zhu ${ }^{1,3}$ and Z. D. Wang ${ }^{1,2,4 *}$ \\ ${ }^{1}$ Department of Physics, University of Hong Kong, Pokfulam Road, Hong Kong, China. \\ ${ }^{2}$ Department of Material Science and Engineering, University of Science and Technology of China, Hefei, China \\ ${ }^{3}$ Department of Physics, South China Normal University, Guangzhou, China \\ 4 Texas Center for Superconductivity, University of Houston, Houston, Tx 7r204
}

\begin{abstract}
We propose an experimentally feasible scheme to achieve quantum computation based on nonadiabatic geometric phase shifts, in which a cyclic geometric phase is used to realize a set of universal quantum gates. Physical implementation of this set of gates is designed for Josephson junctions and for NMR systems. Interestingly, we find that the nonadiabatic phase shift may be independent of the operation time under appropriate controllable conditions. A remarkable feature of the present nonadiabatic geometric gates is that there is no intrinsic limitation on the operation time.
\end{abstract}

PACS numbers: 03.67.Lx, 03.65.Vf, 85.25.Cp

Quantum computers could efficiently solve certain significant problems which are intractable for classical computers [1]. The physical implementation of quantum computation (QC) requires a series of accurately controllable quantum gates. These gates may be implemented experimentally by using controlled dynamic or geometric operations. It is remarkable that geometric operations based on adiabatic passages depend only on the global feature of the path executed [2], and therefore provides a possible fault-tolerant way to perform quantum gate operations [3 6]. Recently, several schemes for adiabatic geometric $\mathrm{QC}$ were proposed by using nuclear magnetic resonance(NMR) [4], trapped ions [5], or superconducting nanocircuits [6]. In particular, an experimental realization of the conditional adiabatic phase shift was reported [4 with the NMR technique. However, the distortions from the adiabatic approximation were also seen in the NMR experiment. Moreover, the adiabatic condition requires that the evolution time must be much longer than the characteristic time $\tau_{0}$ of a qubit system; while the evolution must be completed within the decoherence time. This constraint leads to an intrinsic limitation on the operation time of quantum gates and seems to be a serious obstacle to the physical implementation of some geometric QC schemes, especially for those with solid state systems [6 8], where the decoherence time is very short. Even for the single qubit gate operations based on the adiabatic Berry phase, they might be hard to implement with the current experimental technique [9]. Therefore, it is important to overcome the operation time limitation in geometric $\mathrm{QC}$ set by the adiabatic condition. A twoqubit nonadiabatic geometric phase shift gate was proposed [10] recently for NMR systems. However, it is still an interesting open problem to design a general scheme to achieve the universal set of nonadiabatic geometric gates

\footnotetext{
*To whom correspondence should be addressed. Email address: zwang@hkucc.hku.hk
}

which can be implemented in various physical systems.

In this Letter, we propose a general scheme to achieve the universal set of quantum gates based on cyclic nonadiabatic geometric operations. The present scheme is experimentally feasible, with the required experimental techniques not more stringent than those for dynamical gate operations. Similar to the case with adiabatic passages, the geometric gates based on nonadiabatic cyclic operations also depend only on some global features [11], which make them robust to certain computational errors. Comparing with a nonadiabatic two-qubit gate proposed in Ref.[10], our scheme has at least two distinct advantages: (i) both a two-qubit gate and two noncommutable single qubit gates in a realistic system are designed based on a nonadiabatic geometric method, the latter being also highly nontrivial and useful in QC [5]; (ii) the timeindependent nonadiabatic phase shift may be realized. It is also remarkable that the set of gates designed are not only all-geometric but also all-nonadiabatic, and the scheme is applicable for several potential physical systems.

For universal QC, we need only to achieve two kinds of noncommutable single-qubit gates, and one nontrivial two-qubit gate 12]. Before the design of geometric quantum gates, we show first how to calculate the adiabatic and non-adiabatic geometric phases. For a spin- $1 / 2$ particle subject to an arbitrary magnetic field B, the nonadiabatic cyclic Aharonov-Anandan (AA) phase [11] is just the solid angle determined by the evolution curve in the projective Hilbert space-a unit sphere $S^{2}$. Any two-component 'spin' state $|\psi\rangle=$ $\left[e^{-i \varphi / 2} \cos (\theta / 2), e^{i \varphi / 2} \sin (\theta / 2)\right]^{T}$ may be mapped into a unit vector $\mathbf{n}=(\sin \theta \cos \varphi, \sin \theta \sin \varphi, \cos \theta)$ in the projective Hilbert space via the relation $\mathbf{n}=\langle\psi|\vec{\sigma}| \psi\rangle$, where $T$ represents the transposition of matrix. By changing the magnetic field, the AA phase is given by $\gamma=-\frac{1}{2} \int_{C}(1-\cos \theta) d \varphi$, where $C$ is along the actual evolution curve on $S^{2}$, and is determined by the equation: $\partial_{t} \mathbf{n}(t)=-\mu \mathbf{B}(t) \times \mathbf{n}(t) / \hbar$. This $\gamma$ phase recovers Berry 
phase in adiabatic evolution [13].

We then show how to achieve the universal set of quantum gates based on nonadiabatic geometric AA phases. The single qubit Hamiltonian $\hat{H}$ is chosen to go through a cyclic evolution with period $\tau$ in the parameter space $\{\mathbf{B}\}$. We consider the process where a pair of orthogonal states $\left|\psi_{ \pm}\right\rangle$can evolve cyclically. It is necessary to first decide the above cyclic evolution states. A phase difference between $\left|\psi_{+}\right\rangle$and $\left|\psi_{-}\right\rangle$can be introduced by cyclically changing $\hat{H}$. The phases acquired in this way would contain both a geometric and a dynamical component. The dynamical phase accumulated in the whole process can be removed by a simple method to be described later [4.6, and thus only the geometric phase needs to be considered at present. By taking into account the cyclic condition for $\left|\psi_{ \pm}\right\rangle$and removing the dynamical phase, we have the relation $U(\tau)\left|\psi_{ \pm}\right\rangle=\exp ( \pm i \gamma)\left|\psi_{ \pm}\right\rangle$, where $U(\tau)$ is the evolution operator. Here we have also used the result that $\gamma(-\mathbf{n}(0))=-\gamma(\mathbf{n}(0))$ at any time if the two initial states correspond to $\pm \mathbf{n}(0)$ [13]. We now write an arbitrary initial state as $\left|\psi_{i}\right\rangle=a_{+}\left|\psi_{+}\right\rangle+a_{-}\left|\psi_{-}\right\rangle$with $a_{ \pm}=\left\langle\psi_{ \pm} \mid \psi\right\rangle$, and express the two cyclic initial states as $\left|\psi_{+}\right\rangle=\cos \frac{\chi}{2}|0\rangle+\sin \frac{\chi}{2}|1\rangle$ and $\left|\psi_{-}\right\rangle=-\sin \frac{\chi}{2}|0\rangle+\cos \frac{\chi}{2}|1\rangle$, where $|0\rangle$ and $|1\rangle$ constitute the computational basis for the qubit. The final state at time $\tau$ is found to be $\left|\psi_{f}\right\rangle=U(\chi, \gamma)\left|\psi_{i}\right\rangle$, where

$$
U=\left(\begin{array}{ll}
e^{i \gamma} \cos ^{2} \frac{\chi}{2}+e^{-i \gamma} \sin ^{2} \frac{\chi}{2} & i \sin \chi \sin \gamma \\
i \sin \chi \sin \gamma & e^{i \gamma} \sin ^{2} \frac{\chi}{2}+e^{-i \gamma} \cos ^{2} \frac{\chi}{2}
\end{array}\right) .
$$

It is straightforward to verify that two operations $U^{1}\left(\chi_{1}, \gamma_{1}\right)$ and $U^{2}\left(\chi_{2}, \gamma_{2}\right)$ are noncommutable unless $\sin \gamma_{1} \sin \gamma_{2} \sin \left(\chi_{2}-\chi_{1}\right)=0$. Since two kinds of noncommutable operations constitute a universal set of single-bit gates, we achieve the universal single-bit gates by choosing $\chi_{1} \neq \chi_{2}(\bmod 2 \pi)$ for any nontrivial phases $\gamma_{1}$ and $\gamma_{2}$. For example, the phase-flip gate $U_{1}=\exp \left(-2 i \gamma_{1}|1\rangle\langle 1|\right)$ (up to an irrelevant overall phase) is achieved at $\chi=0$; the gate $U_{2}=\exp \left(i \gamma_{2} \sigma_{x}\right)$ is obtained at $\chi=\pi / 2$, which produces a spin flip (NOT-operation) when $\gamma_{2}=\pi / 2$ and an equal-weight superposition of spin states when $\gamma_{2}=\pi / 4$.

In terms of the computational basis $\{|00\rangle,|01\rangle,|10\rangle$, $|11\rangle\}$, the unitary operator to describe the two-qubit gate is given by $U^{t q}=\operatorname{diag}\left(U_{\left(\gamma^{0}, \chi^{0}\right)}, U_{\left(\gamma^{1}, \chi^{1}\right)}\right)$ under the condition that the control qubit is far away from the resonance condition for the operation of the target qubit. Here $\gamma^{\delta}\left(\chi^{\delta}\right)$ represents the geometric phase (the cyclic initial state) of the target qubit as long as the state of the control qubit corresponds $\delta=0,1$ ( $\delta$ denotes the state of control qubit). Following Ref. [12], we find that the unitary operator $U^{t q}$ is a nontrivial two-qubit gate if and only if $\gamma^{1} \neq \gamma^{0}$ or $\chi^{1} \neq \chi^{0}(\bmod 2 \pi)$. Therefore, all elements of QC may be achievable by using nonadiabatic cyclic geometric operations.
We now describe briefly how to remove the dynamical phase [4,66. We let $\left|\psi_{+/-}\right\rangle$evolve along the time-reversal path of the first-period loop during the second period, namely, the same loop as before is covered backwards by $H_{B}=-\mu \mathbf{B} \cdot \vec{\sigma} / 2$. This process may be simply realized by reversing the effective magnetic field with $\mathbf{B}(2 \tau-t)=$ $-\mathbf{B}(t)$ on the same loop of the first period $[0, \tau)$. We thus have $H_{B}(2 \tau-t)=-H_{B}(t)$ on the loop. As a result, the geometric AA phases accumulated in the two periods will add and the dynamical phase will be cancelled. This is because the dynamical phase $\gamma_{d}^{(2)}$ for the second period has the same magnitude as that $\left(\gamma_{d}^{(1)}\right)$ for the first period but with the opposite sign, i.e., $\gamma_{d}^{(2)}=\int_{\tau}^{2 \tau} d t\left\langle\psi\left|H_{B}(t)\right| \psi\right\rangle$ $=\int_{0}^{\tau} d t\left\langle\psi\left|H_{B}(2 \tau-t)\right| \psi\right\rangle=-\int_{0}^{\tau} d t\left\langle\psi\left|H_{B}(t)\right| \psi\right\rangle=-\gamma_{d}^{(1)}$, where the fact that $|\phi(t)\rangle=|\psi(2 \tau-t)\rangle$ and $|\psi(t)\rangle$ represent the same quantum state is used.

So far, we have proposed a general scheme to achieve nonadiabatic geometric QC. It is important to further consider implementing this scheme with real physical systems. Here, we illustrate this implementation by two examples. The first one is an NMR system [4,14], where the Hamiltonian for a single qubit is given by

$$
H=-\frac{1}{2}\left(\omega_{0} \sigma_{x} \cos \omega t+\omega_{0} \sigma_{y} \sin \omega t+\omega_{1} \sigma_{z}\right),
$$

with $\omega_{i}=\mu B_{i} / h$. Equation (2) can be solved analytically 113. In terms of explicit form of the solution $\mathbf{n}(\chi, \omega t)$ represented in Ref. [13], it is found that the initial states $\left|\psi_{ \pm}\right\rangle$with $\chi=\arctan \left[\omega_{0} /\left(\omega_{1}+\omega\right)\right]$ takes a cyclic evolution with the period $\tau=2 \pi / \omega[10]$, and the evolution path is the curve on a unit sphere swept by the unit vector $\pm \mathbf{n}(\chi, \omega t)$. The corresponding geometric phase is given by $\gamma_{ \pm}= \pm \pi(1-\cos \chi)$ for one cycle. Based on the noncommutable criterion mentioned before, we may use any two processes with different $\omega_{0} /\left(\omega_{1}+\omega\right)$ to achieve two noncommutable qubit gates. The advantage of the above nonadiabatic gates is that there is in principle no limitation on the magnitude of $\omega$. It needs to be noted that, the gates $U_{1,2}$ may not be practical by using the field $\mathbf{B}$ in Eq.(2) since $\gamma=0(\pi)$ as $\chi=0(\pi / 2)$. This problem can be solved by rotating the field. It is seen that the parameter $\chi$ for the initial cyclic state may be changed by rotating the symmetric axis for the field (2)). In the rotated coordinates, $\mathbf{B}^{\prime}=\mathbf{R}\left(\hat{y}, \chi^{\prime}-\chi\right) \mathbf{B}$ (where $\mathbf{R}(\hat{y}, \theta)$ represents the rotation of angle $\theta$ around the $\hat{y}$ axis ) and $\mathbf{n}^{\prime}\left(=\mathbf{R}\left(\hat{y}, \chi^{\prime}-\chi\right) \mathbf{n}(\chi, \omega t)\right)$ because of the spherical symmetry of the system. Thus $\chi$ may change to any required $\chi^{\prime}$ for implementation of the quantum gate, with the geometric phase being unchanged since the area swept by $\mathbf{n}^{\prime}$ is the same as that by $\mathbf{n}$. For example, if the magnetic field is $\mathbf{B}^{\prime}$ for $\chi^{\prime}=\pi / 2$, we may achieve the gate $U_{2}=\exp \left(i \gamma_{2} \sigma_{x}\right)$ with $\gamma_{2}=2 \pi(1-\cos \chi$ ) (where the factor of 2 arises from the evolution of two cycles, which is adopted to remove the dynamic phase). A similar method may be employed to achieve the two-qubit 
operation. The spin-spin interaction in NMR is very well approximated by $H_{I}=J \sigma_{z}^{1} \sigma_{z}^{2} / 2$. We may use the initial state $\left|\psi_{ \pm}\right\rangle$with $\chi^{\delta}=\arctan \left\{\omega_{0} /\left[\omega_{1}+(2 \delta-1) J+\omega\right]\right\}$ to achieve a nonadibatic cyclic two-qubit gate $U^{t q}$. The state of control qubit is (almost) not affected by any operation of the target qubit if $\omega_{1}^{t}$ of the target qubit is chosen to be significantly different from $\omega_{1}^{c}$ of the control qubit (i.e., $\left|\omega_{1}^{t}-\omega_{1}^{c}\right|>>J$ ).

Zhu, Wang, Fig.1

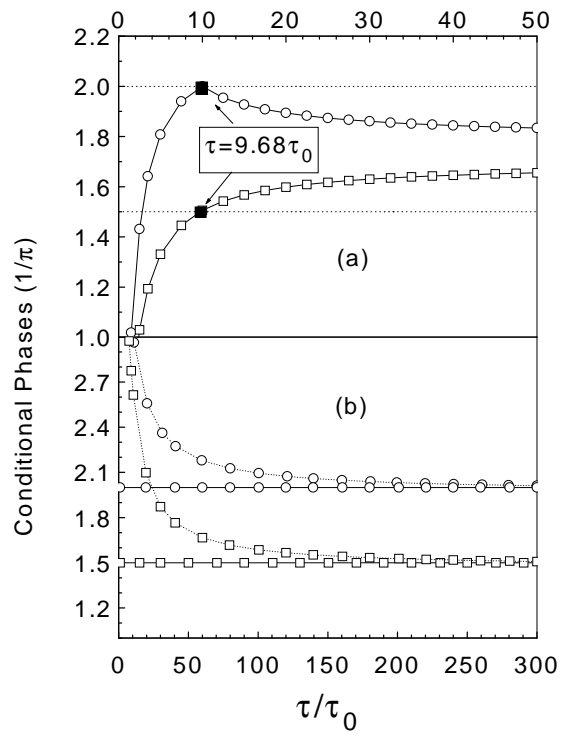

FIG. 1. Conditional geometric phases in an NMR system for (a) $\omega_{1}=0.8 J$ and (b) $\omega_{1}=J-\omega$ with $\omega_{0}=2 \sqrt{15} J$. The solid lines represent actual phases for cyclic evolutions, while the dotted ones are calculated under the adiabatic approximation. Open circles and squares denote $\gamma^{0}$ and $\gamma^{1}$, respectively.

As a typical case, we now show how to achieve the conditional geometric phases for the two-qubit gate $U_{(2 \pi, 3 \pi / 2)}^{t q} \cdot \gamma^{\delta}$ as a function of $\tau / \tau_{0}$ (with $\left.\tau_{0}=2 \pi / \omega_{0}\right)$ is plotted in Fig.1a and Fig.1b, where $\omega_{0}$ is set to be $2 \sqrt{15} J$. First, we assume that $\omega_{1}=\alpha J$ with $0<\alpha<1$, the phase shifts for $U_{(2 \pi, 3 \pi / 2)}^{t q}$ are calculated for $\tau=$ $2 \sqrt{15} /[(1-\alpha) J]$. The curve $\gamma^{\delta} \sim \tau$ is plotted in Fig.1b for $\alpha=0.8$ (the operation time $\tau=5 \sqrt{15} /(2 J)$ ). Secondly, we choose $\omega_{1}=J-\omega$ if $\omega_{1}$ is changeable, then the phase $\gamma^{\delta}$ would be independent on the operation time. The time-independent conditional phase shifts are clearly seen in Fig.1b, namely, the state always evolves along the same closed curve in the projected Hilbert space for the chosen parameters. This also indicates the geometric feature of nonadibatic AA phase. Normally, the state in the projected Hilbert space is controlled by varying the effective field.

The second example is provided by the charge qubit using Josephson junctions, which has been studied in Ref. [6] with adiabatic passages. The generalization to non-adiabatic quantum computation is important for this kind of solid state qubits since the decoherence time in these systems is typically short. The single-qubit(Fig.2a) consists of a superconducting electron box formed by an asymmetric SQUID with the Josephson coupling $E_{1}$ and $E_{2}$, pierced by a magnetic flux $\Phi$ and subject to an applied gate voltage $V_{x}=2 e n_{x}^{e} / C_{x}$ with $2 e n_{x}^{e}$ as the offset charge and $C_{x}$ as the capacitance of the electron box. In the charging regime (where $E_{1,2}<<E_{c h}$ with $E_{c h}$ as charging energy) and at low temperatures, the system behaves as an artificial spin- $1 / 2$ particle in a fictitious magnetic field 15]

$$
\mathbf{B}=\left\{E_{J} \cos \alpha,-E_{J} \sin \alpha, E_{c h}\left(1-2 n_{x}^{e}\right)\right\}
$$

where $E_{J}=\sqrt{E_{-}^{2}+4 E_{1} E_{2} \cos ^{2}\left(\pi \Phi / \Phi_{0}\right)}, \tan \alpha=$ $E_{-} \tan \left(\pi \Phi / \Phi_{0}\right) / E_{+}$with $E_{ \pm}=E_{1} \pm E_{2}$ and $\Phi_{0}=h / 2 e$. By changing $V_{x}$ and $\Phi$, Eq.(3) draws a curve in the parameter space $\{\mathbf{B}\}$. We here study a specific process described by $\pi \Phi(t)=\Phi_{0} \operatorname{atan}\left[E_{+} \tan (\omega t) / E_{-}\right], n_{x}^{e}(t)=$ $\left[1-\left(E_{J} \operatorname{ctg} \chi_{0}+\hbar \omega\right) / E_{c h}\right] / 2$. The fictitious field is a rotating field with a constant frequency(Fig.2b), which guarantees that the angle $\chi_{0}=\arctan \left[E_{J} /\left(B_{z}(t)-\hbar \omega\right)\right]$ is time-independent. We can find that the state denoted by the vector $\mathbf{n}\left(\chi_{0},-\omega t\right)$ undergoes a cyclic evolution with the period $\tau=2 \pi / \omega[10]$. Therefore, we can obtain a unitary operator (1) in the charge-qubit system, where $\chi=\chi_{0}$ and the AA phase $\gamma$ is the half solid angle swept by the vector $\mathbf{n}\left(\chi_{0},-\omega t\right)$.

The interaction between different charge qubits may be realized by coupling two asymmetric SQUIDS capacitively. If the coupling capacitance $C_{I}$ is smaller than the others, the field on the target qubit is given by Eq.(3), but the z-component is replaced by $B_{z}^{\delta}=$ $E_{c h}\left(1-2 n_{x}^{e}\right)+E_{I}\left(n_{x, c}^{e}-\delta\right)$, where $n_{x, c}^{e}$ represents the offset charge in the control qubit and $E_{I}$ is the coupling energy [6]. Obviously, the $\gamma^{\delta}$ phase of the target qubit in the decoupled case is different from $\gamma$ even though $\left(\Phi, n_{x}^{e}\right)$ varies in the same way. If the offset charge $n_{x, c}^{e}$ is timeindependent in the process, the state $\mathbf{n}\left(\chi^{\delta},-\omega t\right)$ with $\chi^{\delta}=\arctan \left[E_{J} /\left(B_{z}^{\delta}-\hbar \omega\right)\right]$ still undergoes a cyclic evolution. Thus the two-qubit operator $U^{t q}$ may be obtained similar to the case in NMR. The elimination of the adiabatic condition for two-bit geometric gates is significant since the coherence time for two qubit gates is typically much shorter than that for single qubit gates. 


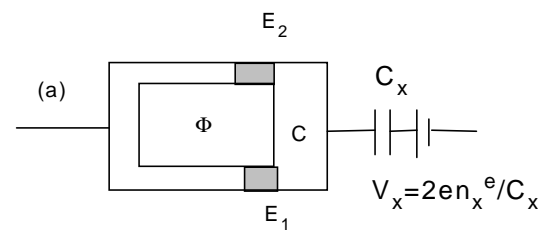

(b)
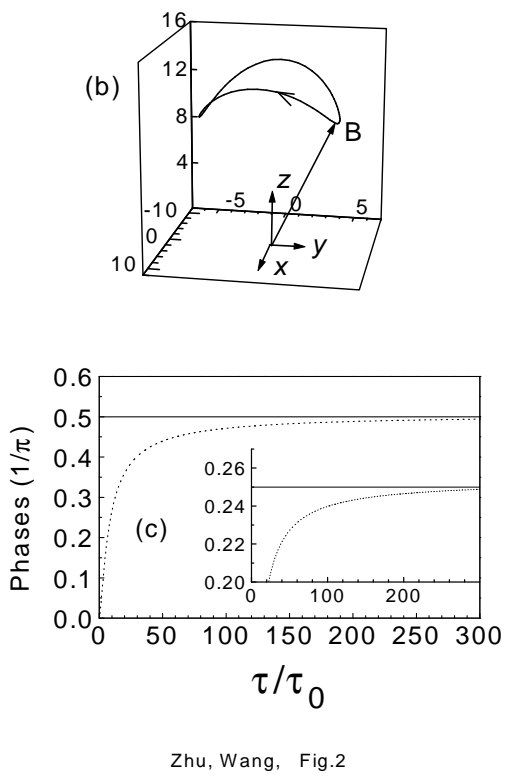

FIG. 2. (a) A schematic Josephson charge qubit(Ref.[6]). (b) The fictitious magnetic field (in unit of $\mu e v$ ) in the designed cyclic process for nonadiabatic geometric gates. (c) Geometric phases to achieve a NOT gate for $E_{2}=4 E_{1}=6.25 \mu e v, E_{c h}=5.0\left(E_{1}+E_{2}\right)$, and $\chi_{0}=\arccos (3 / 4)$. The inset shows the phase shift for the Hadamard operation as $\chi_{0}=\arccos (7 / 8)$. The solid lines represent the actual phases $(\gamma)$ for cyclic evolutions, while the dotted ones $\left(\gamma_{a}\right)$ are calculated under the adiabatic approximation.

To see clearly the advantages of nonadiabatic geometric qubit gates, we compare the operation time between nonadiabatic gates and adiabatic gates, with the phase shift $\pi / 2(\pi / 4)$ corresponding to a NOT (Hadamard) operation. The geometric phases for single-bit operations are shown in Fig. 2c with certain parameters. It is seen from the main panel of Fig. 2c that only if $\tau$ is longer than $70 \tau_{0}\left(=\hbar / E_{J}\right)$, the adiabatic phase $\gamma_{a}$ deviates from the actual nonadiabatic phase $\gamma$ within $10 \%$ error. The similar results can be obtained for other parameters(e.g., see the inset of Fig.2c for $\gamma=\pi / 4$ ). Note that the coherence time achieved by current technology is $30 \sim 40 \tau_{0}$ [B] and thus the theoretical proposal based on the adiabatic phase is not accurate enough to achieve this kind of quantum gate experimentally. In addition, Fig.2c also clearly shows that nonadiabatic phases can be independent of the operation time, similar to the case in NMR systems addressed before.

Finally, we emphasize that the advantages of our scheme are: i.) the designed quantum gates are universal and can handle arbitrary $\mathrm{QC}$ without the intrinsic limitation on operation time; ii.) the scheme is essentially allgeometric and is robust to certain computational errors; iii.) the physical implementation of the scheme can now be realized by realistic Josephson junctions and NMR systems. Nevertheless, it seems to be a limitation of the method that the nonadiabatic phase is more sensitive to fluctuation in the trajectory area than that of the adiabatic phase.

This work was supported by the RGC grant of Hong Kong under Nos. HKU7118/00P and HKU7114/02P, the Ministry of Science and Technology of China under No. G1999064602 and the URC fund of HKU. ZDW thanks support in part from the Texas Center for Superconductivity at the University of Houston. We are very grateful to Drs. L. M. Duan and J. -W. Pan for their valuable suggestions and remarks on the present work. We also thank Prof. C. S. Ting for his critical reading of the manuscript.

[1] P. W. Shor, SIAM Rev. 41, 303 (1999).

[2] M. V. Berry, Proc. R. Soc. London A392, 45 (1984).

[3] P. Zanardi and M. Rasetti, Phys. Lett. A 264, 94 (1999).

[4] J. A. Jones, V. Vedral, A. Ekert, and G. Castagnoli, Nature 403, 869 (2000).

[5] L. M. Duan, J. I. Cirac, and P. Zoller, Science 292, 1695 (2001).

[6] G. Falci, R. Fazio, G. M. Palma, J. Siewert, and V. Vedral, Nature 407, 355 (2000).

[7] Y. Makhlin, G. Schön, and A. Shnirman, Rov. Mod. Phys. 73, 357 (2001).

[8] Y. Nakamura, Yu. A. Pashkin, and J. S. Tsai, Nature 398, 786 (1999).

[9] S. L. Zhu and Z. D. Wang, Physica C 364, 213 (2001).

[10] X. B. Wang and M. Keiji, Phys. Rev. Lett. 87, 097901 (2001); 88, 179901(E) (2002).

[11] Y. Aharonov and J. Anandan, Phys. Rev. Lett. 58, 1593 (1987).

[12] S. Lloyd, Phys. Rev. Lett. 75, 346 (1995).

[13] S. L. Zhu and Z. D. Wang, Phys. Rev. Lett. 85, 1076 (2000).

[14] N. Gershenfeld and I. L. Chuang, Science 275, 350 (1997).

[15] D. V. Averin and K. K. Likharev, in B. L. Altshuler, P. A. Lee, and R. A. Webb, Mesoscopic Phenomena in Solids (Elsevier, New York, 1991), p213. 